\title{
LETTRE : UTILISATION CONJOINTE DE SÉRIES TEMPORELLES D'IMAGES OPTIQUES ET RADAR POUR LE SUIVI DES SURFACES AGRICOLES
}

\author{
Jordi Inglada \\ CESBIO, Université de Toulouse, CNES/CNRS/IRD/UPS, Toulouse, France
}

Dans un contexte de pression croissante sur les terres agricoles et leur production, Foley et al. (2011), Godfray et al. (2010), la cartographie des cultures est nécessaire pour fournir des informations précises pour leur conduite efficace et durable. L'imagerie de télédétection en général, et plus particulièrement les séries temporelles d'images à haute résolution comme celles fournies par les satellites Sentinel-1, Torres et al. (2012), et Sentinel-2, Drusch et al. (2012), sont un atout majeur pour ce type d'applica-tion.

Des travaux récents ont montré que les séries temporelles d'imagerie optique permettent de cartographier de façon précise les surfaces agricoles dans différents types de climat et des systèmes de cultures variés, Inglada et al. (2015). Cependant, dû à la couverture nuageuse qui affecte l'imagerie optique, les performances de ces approches peuvent être fortement dégradées dans certains cas. L'imagerie radar, insensible aux nuages, peut aider à la mise en oeuvre de méthodes de cartographie plus robustes.

Au delà de la cartographie annuelle comme celle abordée dans Inglada et al. (2015), la détection précoce des cultures avant la fin de la saison agricole est nécessaire pour la prévision des rendements et la gestion de l'irrigation. Ceci impose des contraintes encore plus fortes sur la disponibilité d'imagerie multi-temporelle.

Nous illustrons ici comment l'imagerie radar peut complémenter les séries temporelles optiques pour la détection précoce des cultures dans une perspective d'utilisation conjointe des données des satellites Sentinel-1 et Sentinel-2. Nous nous intéressons particulièrement à la classification incrémentale produite au fur et à mesure que les images sont acquises.

En attendant la disponibilité d'images Sentinel-2 sur une saison agricole complète (le satellite est entré en phase opérationnelle en mars 2016), l'étude a été menée avec des images du satellite Landsat 8 , qui a des caractéristiques similaires (revisite de 16 jours au lieu de 5 avec 2 satellites Sentinel-2, et $30 \mathrm{~m}$ de résolution au lieu de 10). Les images radar utilisées sont issues du premier satellite Sentinel-1. En tout, 11 images Landsat 8 et 9 images Sentinel-1 d'une zone agricole au SudOuest de Toulouse ont été utilisées.

La figure 1 synthétise les résultats de la classification incrémentale pour 3 scénarios :

\footnotetext{
1. l'utilisation de l'imagerie optique seule (bleu);

2. l'utilisation de l'imagerie radar seule (rouge);

3. l'utilisation conjointe des 2 modalités (jaune).
}

Le graphique présente la valeur moyenne de l'indice $\mathrm{K}$ (mesure de qualité de la classification allant de 0 à 1) sur 10 réalisations et les intervalles de confiance à $95 \%$. On peut observer le motif attendu de l'amélioration des performances au fur et à mesure que plus de données sont disponibles. Ce comportement est le même pour les 3 scénarios. On peut aussi constater que l'utilisa-tion d'imagerie optique fournit de meilleurs résultats que l'utilisation d'imagerie radar. Ceci n'est pas une surprise, car l'information principale permettant de distinguer les cultures est le profil temporel de croissance de la végétation, qui est très bien capturé par le NDVI. Pendant une courte période, en fin d'automne, le radar, sensible aux changements de rugosité liés aux travaux du sol, fournit de meilleurs résultats.

L'utilisation conjointe des 2 types d'imagerie produit toujours de meilleurs résultats que chaque source de données utilisée indépendamment. II est aussi intéressant de noter que, début avril, la fusion des 2 sources de données atteint des niveaux de performance qui ne seront obtenus par l'imagerie optique seule qu'un mois plus tard. Ceci constitue un gain non négligeable pour la détection précoce des cultures.

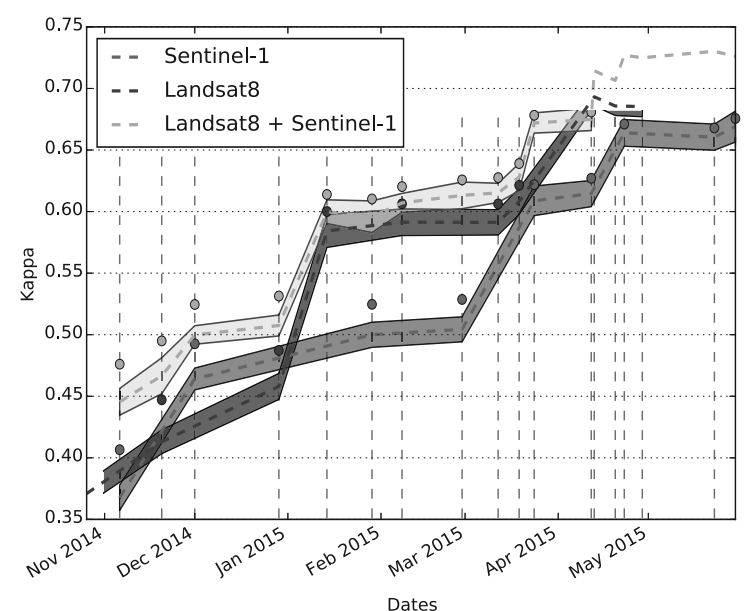

FIGURE 1 : Mesure de performances de la classification incrémentale des cultures agricoles pour les 3 scénarios étudiés. La largeur des traits correspond aux intervalles de confiance à 95\%

La figure 2 illustre des extraits de cartes d'occupation des sols obtenues avec l'ensemble des images disponibles. La carte issue des images radar seules est plus bruitée que celles obtenues en utilisant des données optiques; mais globalement, les cartes sont cohérentes. 

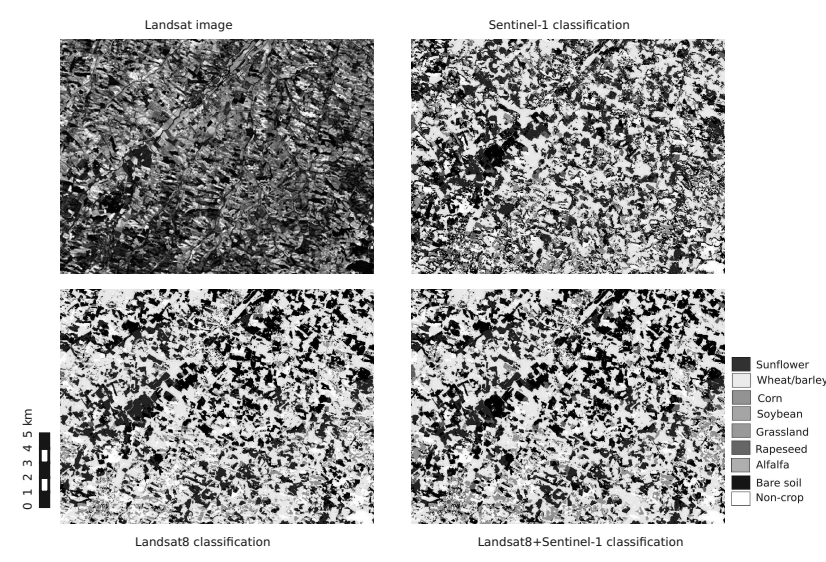

FIGURE 2: Extraits de cartes de type de cultures avec toutes les images disponibles. Image Landsat8 (haut à gauche), carte issue du radar (haut à droite), carte issue des données optiques (bas à gauche) et carte issue de la combinaison des 2 types de données (bas à droite).

On peut conclure que les images à haute résolution spatiale et temporelle fournies par Sentinel-1 sont un bon complément à celles des systèmes optiques (Landsat 8 et Sentinel-2) pour la reconnaissance précoce des types de cultures. Les données radar permettent une amélioration des performances de classification plus tôt dans la saison agricole par rapport au cas où seulement l'imagerie optique est utilisée.

Pour une étude plus détaillée portant sur l'extraction de primitives issues de l'imagerie radar ainsi que sur les choix des pré-traitements des données, le lecteur intéressé peut se référer à des résultats présentés dans Inglada et al. (2016). Dans cet article en accès ouvert, différents choix de résolution de travail et de filtrage du " speckle " sont comparés dans un contexte de classification pixel en utilisant des forêts aléatoires (Random Forests). En effet, les images Sentinel-1 ayant une résolution plus fine que celle des images Landsat-8, il est important de choisir de travailler à la résolution native, ou à celle du capteur de référence pour ces travaux. On y conclut que ré-échantillonner les images Sentinel-1 à la résolution de $30 \mathrm{~m}$ de Landsat-8 produit des résultats équivalents (en termes de précision de classification) à travailler à pleine résolution. En revanche, si un filtre anti-speckle est utilisé (filtre de Lee dans cette étude), ré-échantillonner toutes les images à $10 \mathrm{~m}$ de résolution fournit des résultats meilleurs en termes de précision de classification tout en fournissant un détail géométrique supérieur.

\section{Références}

Drusch, M., Bello, U. D., Carlier, S., Colin, O., Fernandez, V., Gascon, F., Hoersch, B., Isola, C., Laberinti, P., Martimort, P., Meygret, A., Spoto, F., Sy, O., Marchese, F., Bargellini, P., 2012. Sentinel-2 : Esa's optical high-resolution mission for Gmes operational services. Remote Sensing of Environment 120 (nil), 25-36.

URL http://dx.doi.org/10.1016/j.rse.2011.11.026
Foley, J. A., Ramankutty, N., Brauman, K. A., Cassidy, E. S., Gerber, J. S., Johnston, M., Mueller, N. D., O'Connell, C., Ray, D. K., West, P. C., Balzer, C., Bennett, E. M., Carpenter, S. R., Hill, J., Monfreda, C., Polasky, S., Rockström, J., Sheehan, J., Siebert, S., Tilman, D., Zaks, D. P. M., 2011. Solutions for a cultivated planet. Nature 478 (7369), 337-342.

URL http://dx.doi.org/10.1038/nature10452

Godfray, H. C. J., Beddington, J. R., Crute, I. R., Haddad, L., Lawrence, D., Muir, J. F., Pretty, J., Robinson, S., Thomas, S. M., Toulmin, C., 2010. Food security : The challenge of feeding 9 billion people. Science 327 (5967), 812-818. URL http://dx.doi.org/10.1126/science. 1185383

Inglada, J., Arias, M., Tardy, B., Hagolle, O., Valero, S., Morin, D., Dedieu, G., Sepulcre, G., Bontemps, S., Defourny, P., Koetz, B., 2015. Assessment of an operational system for crop type map production using high temporal and spatial resolution satellite optical imagery. Remote Sensing 7 (9), 12356-12379.

URL http://dx.doi.org/10.3390/rs70912356

Inglada, J., Vincent, A., Arias, M., Marais-Sicre, C., 2016. Improved early crop type identification by joint use of high temporal resolution sar and optical image time series. Remote Sensing 8 (5), 362.

URL http://dx.doi.org/10.3390/rs8050362

Torres, R., Snoeij, P., Geudtner, D., Bibby, D., Davidson, M., Attema, E., Potin, P., Rommen, B., Floury, N., Brown, M., Traver, I. N., Deghaye, P., Duesmann, B., Rosich, B., Miranda, N., Bruno, C., L'Abbate, M., Croci, R., Pietropaolo, A., Huchler, M., Rostan, F., 2012. Gmes sentinel-1 mission. Remote Sensing of Environment 120 (nil), 9-24. URL http://dx.doi.org/10.1016/j.rse.2011.05.028 